Research Paper

\title{
Association of Maternal Serum 25-hydroxyvitamin D Concentrations in Second Trimester with Delivery Mode in A Chinese Population
}

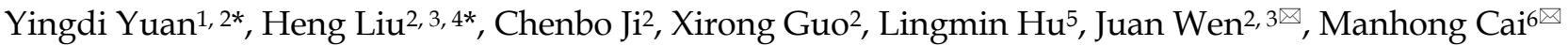 \\ 1. The First People's Hospital of Lianyungang, Xuzhou Medical University Affiliated Hospital of Lianyungang, Lianyungang, 222002, China; \\ 2. Nanjing Maternity and Child Health Care Institute, Nanjing Maternity and Child Health Care Hospital, Obstetrics and Gynecology Hospital Affiliated to \\ Nanjing Medical University, Nanjing, 210004, China; \\ 3. State key Laboratory of Reproductive Medicine, Nanjing Maternity and Child Health Care Hospital, Obstetrics and Gynecology Hospital Affiliated to \\ Nanjing Medical University, Nanjing, 210004, China; \\ 4. Department of Pediatrics, Nanjing Maternity and Child Health Care Hospital, Obstetrics and Gynecology Hospital Affiliated to Nanjing Medical University, \\ Nanjing, 210004, China; \\ 5. Changzhou Maternity and Child Health Care Hospital affiliated Nanjing Medical University, Changzhou, 213003, China; \\ 6. Department of Obstetrics, Nanjing Maternity and Child Health Care Hospital, Obstetrics and Gynecology Hospital Affiliated to Nanjing Medical \\ University, Nanjing, 210004, China. \\ * These authors contributed equally to this work. \\ $\square$ Corresponding authors: Juan Wen (wenj2010@gmail.com) and Manhong Cai (manhongcai@126.com)
}

(c) Ivyspring International Publisher. This is an open access article distributed under the terms of the Creative Commons Attribution (CC BY-NC) license (https://creativecommons.org/licenses/by-nc/4.0/). See http://ivyspring.com/terms for full terms and conditions.

Received: 2017.03.30; Accepted: 2017.07.05; Published: 2017.08.18

\begin{abstract}
Objective: To determine the maternal serum 25-hydroxyvitamin $\mathrm{D}[25(\mathrm{OH}) \mathrm{D}]$ concentrations in a Chinese population and investigate its associations with subsequent delivery mode by studying 1924 unrelated pregnant women.

Methods: The serum 25(OH)D concentrations was measured by euzymelinked immunosorbent assay (ELISA). Simultaneously, maternal information and subsequent delivery mode were collected. Logistic regression analysis was performed to assess the associations between $25(\mathrm{OH}) \mathrm{D}$ concentrations and caesarean section.

Results: The median (IQR) serum concentration of 25(OH)D for the total subjects was 43.4 $(35.2-56.9) \mathrm{nmol} / \mathrm{L}$. Among them, 1225 (63.7\%) women were in the status of $25(\mathrm{OH}) \mathrm{D}$ deficiency $(<$ $50.0 \mathrm{nmol} / \mathrm{L})$. The $25(\mathrm{OH}) \mathrm{D}$ concentrations showed significant variation by body mass index (BMI), parity and season of sampling. Women with caesarean section was older, and with higher BMI and rate of abnormal pregnancy history, suggesting advanced age, obesity and abnormal pregnancy history may be the risk factors for the subsequent caesarean section. Compared with 25(OH)D from 50.0 to 74.9 $\mathrm{nmol} / \mathrm{L}$, women with low $25(\mathrm{OH}) \mathrm{D}$ concentrations $(<50.0 \mathrm{nmol} / \mathrm{L})$ was not significantly associated with caesarean section. Only in the subgroup of the women without abnormal pregnancy history, higher 25(OH)D (> $75.0 \mathrm{nmol} / \mathrm{L})$ concentrations could significantly decrease the risk of caesarean section.

Conclusion: Vitamin $D$ deficiency is a quite serious problem in Chinese pregnant women. There is no evidence that the maternal serum $25(\mathrm{OH}) \mathrm{D}$ concentrations is associated with increased risk of caesarean section.
\end{abstract}

Key words: 25(OH)D; Vitamin D deficiency; Caesarean section.

\section{Introduction}

In recent years, a large number of people in different areas were in status of vitamin D deficiency or insufficiency, which has been a globally and gradually serious problem [1]. Vitamin D is a lipid soluble vitamin indispensable to human body. The main function of active vitamin $\mathrm{D}$ is to increase the intestinal absorption of calcium, regulate the function of bone cells, affect bone metabolism, and to maintain bone health at all ages [2,3]. An increasing number of studies have shown that vitamin $\mathrm{D}$ deficiency in 
serum during pregnancy are closely related to a series of adverse pregnancy outcomes, including gestational diabetes, preeclampsia, preterm birth, intrauterine growth restriction, and so on [4-6]. Despite lack of vitamin $\mathrm{D}$ has indeed affected human health, how to define lack of vitamin $\mathrm{D}$ is still an academic controversy $[7,8]$. 25-Hydroxyvitamin $\mathrm{D}[25(\mathrm{OH}) \mathrm{D}]$ is the storage form of vitamin $\mathrm{D}$, which is an ideal indicator of vitamin D levels [9]. Most scholars defined vitamin $\mathrm{D}$ deficiency as serum $25(\mathrm{OH}) \mathrm{D}$ concentrations < $50.0 \mathrm{nmol} / \mathrm{L}(20.0 \mathrm{ng} / \mathrm{mL})$, and defined vitamin $D$ insufficiency as serum $25(\mathrm{OH}) \mathrm{D}$ concentrations $<75.0 \mathrm{nmol} / \mathrm{L}(30.0 \mathrm{ng} / \mathrm{mL})$ [10]. But it is not clear that the proposed $25(\mathrm{OH}) \mathrm{D}$ concentrations are suitable for pregnant women.

With the rapid development of the technique and method of cesarean section, cesarean section has been one of the most commonly used surgical procedures, which is an effective rescue measure to solve the dystocia and some obstetric syndrome of pregnant women [11]. So in many regions, the application of cesarean section has increased substantially over recent decades. However, its disadvantages are also prominent. The pregnant women are prone to bleeding, infection, organ adhesion, and prone to be affected with some other short or long-term complications, whereas the newborns are prone to temporary tachypnea, pathological jaundice, and so on [12, 13]. An observational study from Boston shown that the cesarean section rate in pregnant women with serum $25(\mathrm{OH}) \mathrm{D}<37.5 \mathrm{nmol} / \mathrm{L}$ was over 4 times more than women with normal 25(OH)D concentrations [14]. However, Bowyer et al. had come to a different conclusion that there is no correlation between vitamin $\mathrm{D}$ levels during pregnancy and delivery mode [15]. Currently, there are few studies on the relationship between the level of maternal vitamin $\mathrm{D}$ and the mode of delivery. In this study, we measured the serum $25(\mathrm{OH}) \mathrm{D}$ concentrations in 1924 pregnant women in second trimester to describe the current situation of vitamin D deficiency in Chinese pregnant women, and to evaluate the relationship between vitamin $\mathrm{D}$ levels and delivery mode.

\section{Materials and Methods}

This study was approved by the ethics committee of Nanjing Medical University, and the experimental methods were performed strictly in accordance with the approved guidelines.

\section{Study subjects}

The pregnant women of this study were recruited from March 2012 to February 2015 in Obstetrics and Gynecology Hospital Affiliated to
Nanjing Medical University (between 31 and 32 degrees north latitude), and the related information were collected through questionnaires and conventional electronic medical records. The exclusion criteria included loss of basic information, medical abortion, multiple pregnancy, and the use of assisted reproductive technology or $25(\mathrm{OH}) \mathrm{D}$ concentrations beyond the assay detection limit. Each pregnant woman was collected $5 \mathrm{ml}$ peripheral blood after completing the questionnaire, and the serum was separated by centrifugation at the Nanjing Maternal and Child Health Institute (NMCHI). Then the samples were stored in chronological order in the 100 -hole boxes at $-80^{\circ} \mathrm{C}$. Using the random number table, the 100-hole boxes were randomly selected from the total population. All cesarean section samples in one box were defined as the case group, and the others were defined as the control group. In the end, 1924 pregnant women agreed to participate in the study and signed informed consents.

\section{Vitamin D measurement}

After serum sample thawed, 25(OH)D concentrations was determined by using an euzymelinked immunosorbent assay (ELISA) (25-hydroxy vitamin D Kit, IDS Ltd, Boldon, UK). The inter- and intra-assay coefficients of variation were $4.6 \%$ and $5.3 \%$, respectively. And the reported analytic sensitivity of the assay was from 6.8 to 380 $\mathrm{nmol} / \mathrm{L}$. For the measurement of serum $25(\mathrm{OH}) \mathrm{D}$, the liquid chromatography-tandem mass spectrometry (LC-MS/MS) method is generally considered to be the best way, however, in our study, the enzyme immunoassay was used. Although the immunoassay may result in a little negative biases and misclassification of participants for vitamin D sufficiency when compared with the LC-MS/MS assay, it is more readily available and require small volume sampling, ideal for testing. All the samples were tested by trained researchers who were not aware of the source of the samples. The commonly used cutoff values for $25(\mathrm{OH}) \mathrm{D}$ status were $25,37.5$, 50 and $75 \mathrm{nmol} / \mathrm{L}$ [16].

\section{Data source}

After the investigators (epidemiology professionals) were trained qualified, a unified designed and pre-investigated questionnaire was used to collected related information. The questionnaire included demographic data, menarche age, menstrual cycle, previously diagnosed hypertension (chronic or pregnancy) or diabetes (pre-gestational or gestational), family history of hypertension or diabetes, history of uterine fibroids, adverse pregnancy history, and so on. The 
information of subsequent delivery mode (cesarean section or vaginal delivery) was obtained from routine electronic medical record.

\section{Statistical analysis}

All the investigation data and experimental data were examined and verified. The EpiData 3.02 software was used to input data and establish the database. Percentiles were used to describe the distribution of $25(\mathrm{OH}) \mathrm{D}$ concentrations in the total pregnant women population and its subgroups. Kruskal-Wallis or Mann-Whitney test was used to analyze the difference of the distribution of 25(OH)D concentrations in each subgroup. Student's $t$-test, $X^{2}$ test or Mann-Whitney test were used to compare the demographic characteristics and the distribution of 25(OH)D $\quad(<25, \quad 25-37.4,37.5-49.9, \quad 50-74.9$, > $75 \mathrm{nmol} / \mathrm{L})$ between cesarean section and spontaneous delivery group, and univariate and multivariate logistic regression analysis were performed to evaluate the crude and adjusted association between $25(\mathrm{OH}) \mathrm{D}$ concentrations and delivery mode by computing odds ratios (ORs) and 95\% confidence interval (CIs). The adjustment factors including maternal age, intrapartum body mass index (BMI), birthplace, parity, menarche age, menstrual cycle, abnormal pregnancy history, sampling season, previously diagnosed hypertension or diabetes, family history of hypertension or diabetes, and history of uterine fibroids. All the above statistical analyses were performed with SPSS 18.0 software, and $P \leq 0.05$ for a two-sided test was considered statistically significant.

\section{Results}

In total, 2318 serum samples were tested for 25(OH)D, of which 2131 (91.9\%) pregnant women had detailed health information. We excluded 207 women who had medical abortion or multiple pregnancy, who applied assisted reproductive technology, or who had $25(\mathrm{OH}) \mathrm{D}$ concentrations exceeded the assay detection limit. In the end, 1924 pregnant women were included in the study. The percentile distributions of $25(\mathrm{OH}) \mathrm{D}$ in the total women and subgroups were shown in Table 1 . In the total population, the median (IQR) concentration of 25(OH)D was $43.4(35.2$ - 56.9) nmol/L; 53 pregnant women $(2.8 \%)$ had concentrations < $25.0 \mathrm{nmol} / \mathrm{L} ; 567$ $(29.5 \%)$ had concentrations from 25.0 to $37.4 \mathrm{nmol} / \mathrm{L}$; 605 pregnant women $(31.4 \%)$ had concentrations from 37.5 to $49.9 \mathrm{nmol} / \mathrm{L} ; 492(25.6 \%)$ had concentrations from 50.0 to $74.9 \mathrm{nmol} / \mathrm{L} ; 207$ pregnant women $(10.8 \%)$ had concentrations $>75.0 \mathrm{nmol} /$ L. Pregnant women with intrapartum BMI $\geq 30 \mathrm{~kg} / \mathrm{m}^{2}$ and multipara had lower $25(\mathrm{OH}) \mathrm{D}$ concentrations $(P=$ 0.015 and 0.010 , respectively), and there was a significant variation of $25(\mathrm{OH}) \mathrm{D}$ concentrations by sampling season $(P<0.001)$. The 25(OH)D concentrations was relatively higher in summer and autumn, and lower in spring and winter, suggesting that vitamin D levels were closely related to sunlight exposure. However, in other subgroups (by maternal age, birthplace, menarche age, menstrual cycle and adverse pregnancy history), no significant differences in the $25(\mathrm{OH}) \mathrm{D}$ concentrations were observed (all $P>$ 0.05).

In this study, there were 1883 pregnant women with delivery information, including 1060 cases of spontaneous labor and 823 cases of cesarean section. As shown in table 2, pregnant women with cesarean section were more likely to be older, to have higher intrapartum BMI, and more likely to have an abnormal pregnancy history as compared with controls (all $P<0.05$ ). In addition, pregnant women with cesarean section were less likely to be nullipara with a borderline significance $(P=0.060)$. These evidences suggested advanced age, obesity, abnormal pregnancy history and multipara may be the risk factors for the subsequent caesarean section. However, there was no significant difference in birthplace, menarche age, menstrual cycle, sampling season, 25(OH)D concentrations and distributions between the cesarean section group and controls (all $P$ $>0.05$ ).

Univariate and multivariate logistic regression and subgroup analysis showed that there was no association between low 25(OH)D concentrations (< $50.0 \mathrm{nmol} / \mathrm{L}$ ) and risk of cesarean section, as compared with women with $25(\mathrm{OH}) \mathrm{D}$ concentrations from 50.0 to $74.9 \mathrm{nmol} / \mathrm{L}$. And the results of the subgroup analysis were consistent with the results of the total population. While for the higher $25(\mathrm{OH}) \mathrm{D}$ concentrations $(>75.0 \mathrm{nmol} / \mathrm{L})$, univariate and multivariate logistic regression analysis also suggested no significant association between the $25(\mathrm{OH}) \mathrm{D}$ concentrations and risk of cesarean section. Only in the subgroup of the women without abnormal pregnancy history, higher 25(OH)D concentrations (> $75.0 \mathrm{nmol} / \mathrm{L})$ could significantly decrease the risk of caesarean section $(\mathrm{OR}=0.65$, $95 \% \mathrm{CI}=0.45-0.96$ ). After adjustment for confounding factors, a borderline significant protective effect of higher $25(\mathrm{OH}) \mathrm{D}$ concentrations for caesarean section was also observed $(\mathrm{OR}=0.70$, $95 \% \mathrm{CI}=0.47-1.05)$ (Table 3). 
Table 1. The $25(\mathrm{OH}) \mathrm{D}$ concentrations by maternal characteristics

\begin{tabular}{|c|c|c|c|c|c|c|c|c|c|}
\hline \multirow[t]{3}{*}{ Maternal characteristics } & \multirow[t]{3}{*}{$n(\%)$} & \multicolumn{7}{|c|}{$25(\mathrm{OH}) \mathrm{D}$ percentiles } & \multirow[t]{3}{*}{$P$ a } \\
\hline & & 5 th & 10th & 25th & 50 th & 75th & 90th & 95th & \\
\hline & & \multicolumn{7}{|c|}{$n m o l / L$} & \\
\hline All women & $1924(100.0)$ & 26.6 & 29.5 & 35.2 & 43.4 & 56.9 & 77.0 & 94.9 & - \\
\hline \multicolumn{10}{|l|}{ Maternal age (year) } \\
\hline$<25$ & $92(4.9)$ & 25.6 & 28.9 & 35.6 & 43.7 & 56.4 & 79.6 & 101.5 & \multirow[t]{5}{*}{0.261} \\
\hline $25-$ & $915(48.6)$ & 26.6 & 29.2 & 35.0 & 43.0 & 55.7 & 74.4 & 93.0 & \\
\hline $30-$ & $731(38.8)$ & 26.8 & 30.0 & 35.9 & 44.2 & 61.0 & 78.1 & 94.8 & \\
\hline $35-$ & $124(6.6)$ & 25.1 & 29.6 & 34.3 & 42.9 & 55.4 & 80.9 & 106.3 & \\
\hline $40-$ & $21(1.1)$ & 24.8 & 27.4 & 34.5 & 44.1 & 61.1 & 70.1 & 159.2 & \\
\hline \multicolumn{10}{|l|}{ Intrapartum BMI $\left(\mathrm{kg} / \mathrm{m}^{2}\right)$} \\
\hline$<25$ & $541(29.1)$ & 27.0 & 30.3 & 35.7 & 44.8 & 59.2 & 80.8 & 97.0 & \multirow[t]{4}{*}{0.015} \\
\hline $25-$ & 995 (53.4) & 26.6 & 29.4 & 35.6 & 43.0 & 57.5 & 77.1 & 93.9 & \\
\hline $30-$ & $281(15.1)$ & 24.8 & 27.7 & 34.2 & 40.4 & 52.8 & 69.1 & 93.4 & \\
\hline $35-$ & $45(2.4)$ & 25.6 & 30.2 & 34.6 & 44.7 & 51.6 & 71.7 & 82.5 & \\
\hline \multicolumn{10}{|l|}{ Birthplace } \\
\hline Jiangsu province & $1819(96.7)$ & 26.6 & 29.5 & 35.4 & 43.5 & 57.1 & 76.9 & 95.0 & \multirow[t]{2}{*}{0.762} \\
\hline Other provinces & $63(3.3)$ & 26.0 & 27.6 & 33.4 & 41.9 & 61.0 & 81.7 & 92.0 & \\
\hline \multicolumn{10}{|l|}{ Parity } \\
\hline Nullipara & $1777(93.4)$ & 26.7 & 29.5 & 35.6 & 43.8 & 57.4 & 77.7 & 95.3 & \multirow[t]{2}{*}{0.010} \\
\hline Multipara & $126(6.6)$ & 26.0 & 29.0 & 33.4 & 39.8 & 51.4 & 62.9 & 85.7 & \\
\hline \multicolumn{10}{|l|}{ Menarche age (year) } \\
\hline$<13$ & $314(16.7)$ & 26.3 & 29.2 & 35.9 & 44.8 & 60.9 & 81.3 & 99.0 & \multirow[t]{3}{*}{0.154} \\
\hline 13- & $1237(65.7)$ & 27.0 & 29.7 & 35.5 & 43.5 & 56.8 & 75.8 & 94.2 & \\
\hline $15-$ & 332 (17.6) & 25.8 & 28.0 & 33.7 & 42.2 & 56.4 & 76.4 & 91.2 & \\
\hline \multicolumn{10}{|l|}{ Menstrual cycle (day) } \\
\hline $21-$ & $1656(88.0)$ & 26.5 & 29.3 & 35.2 & 43.5 & 57.2 & 77.7 & 96.5 & \multirow[t]{3}{*}{0.945} \\
\hline 36- & $162(8.6)$ & 26.6 & 29.8 & 35.4 & 43.0 & 58.8 & 73.6 & 86.9 & \\
\hline Irregularity & $64(3.4)$ & 28.4 & 30.8 & 36.9 & 43.5 & 52.8 & 66.8 & 79.4 & \\
\hline \multicolumn{10}{|c|}{ Abnormal pregnancy history } \\
\hline No & $1578(82.0)$ & 26.8 & 29.5 & 35.2 & 43.4 & 56.8 & 77.0 & 95.3 & \multirow[t]{2}{*}{0.878} \\
\hline Yes & $346(18.0)$ & 25.9 & 29.2 & 35.2 & 43.6 & 57.6 & 76.9 & 91.0 & \\
\hline \multicolumn{10}{|l|}{ Sampling season } \\
\hline Spring & $553(28.7)$ & 24.7 & 26.7 & 32.7 & 40.8 & 53.2 & 67.8 & 83.8 & \multirow[t]{4}{*}{$<0.001$} \\
\hline Summer & $632(32.8)$ & 29.7 & 32.0 & 37.1 & 46.0 & 59.6 & 79.8 & 95.6 & \\
\hline Autumn & $361(18.8)$ & 28.4 & 30.7 & 36.1 & 44.6 & 61.0 & 93.5 & 106.3 & \\
\hline Winter & $378(19.6)$ & 26.3 & 28.9 & 35.1 & 42.5 & 56.0 & 69.6 & 88.0 & \\
\hline
\end{tabular}

a $P$-values were determined by using the Kruskal-Wallis or Mann-Whitney test. Abbreviations: 25(OH)D, 25-hydroxyvitamin D; BMI, body mass index.

Table 2. Maternal characteristics and $25(\mathrm{OH}) \mathrm{D}$ serum concentrations between women with natural labor and cesarean section

\begin{tabular}{|c|c|c|c|}
\hline \multirow[t]{2}{*}{ Maternal characteristics } & Natural labor & Cesarean section & \multirow[t]{2}{*}{$P \mathrm{~b}$} \\
\hline & $(\mathrm{n}=1060)$ & $(\mathrm{n}=823)$ & \\
\hline Maternal age (years) a & $28.9 \pm 3.0$ & $30.2 \pm 3.8$ & $<0.001$ \\
\hline Intrapartum BMI $\left(\mathrm{kg} / \mathrm{m}^{2}\right)$ a & $26.3 \pm 3.1$ & $27.9 \pm 3.6$ & $<0.001$ \\
\hline Birthplace of Jiangsu Province [n (\%)] & $1024(96.6)$ & 795 (96.7) & 0.894 \\
\hline Nullipara [n (\%)] & $1013(94.3)$ & $764(92.2)$ & 0.060 \\
\hline Menarche age a & $13.6 \pm 1.1$ & $13.6 \pm 1.3$ & 0.283 \\
\hline Irregular menstrual cycle [n (\%)] & $31(2.9)$ & $33(4.0)$ & 0.196 \\
\hline Having abnormal pregnancy history [n (\%)] & $145(13.2)$ & $201(24.2)$ & $<0.001$ \\
\hline Sampling in summer and autumn [n (\%)] & $577(52.7)$ & $416(50.2)$ & 0.275 \\
\hline $25(\mathrm{OH}) \mathrm{D}(\mathrm{nmol} / \mathrm{L}) \mathrm{c}$ & $43.0(35.2,57.4)$ & $43.4(35.3,56.4)$ & 0.950 \\
\hline \multicolumn{4}{|l|}{$25(\mathrm{OH}) \mathrm{D}[\mathrm{n}(\%)]$} \\
\hline$<25.0 \mathrm{nmol} / \mathrm{L}$ & $29(2.6)$ & $24(2.9)$ & 0.542 \\
\hline $25.0-37.4 \mathrm{nmol} / \mathrm{L}$ & $330(30.1)$ & 237 (28.6) & \\
\hline $37.5-49.9 \mathrm{nmol} / \mathrm{L}$ & 338 (30.9) & $267(32.2)$ & \\
\hline $50.0-74.9 \mathrm{nmol} / \mathrm{L}$ & $271(24.7)$ & $221(26.7)$ & \\
\hline$>75 \mathrm{nmol} / \mathrm{L}$ & 127 (11.6) & $80(9.7)$ & \\
\hline
\end{tabular}

a Mean \pm SD; ${ }^{b} P$-values were determined by using $t$ test, $X^{2}$ test or Mann-Whitney test; c Median (IQR). Abbreviations: 25(OH)D, 25-hydroxyvitamin D; BMI, body mass index. 
Table 3. Association between $25(\mathrm{OH}) \mathrm{D}$ serum concentrations and cesarean section and subgroup analysis

\begin{tabular}{|c|c|c|c|c|c|c|c|c|c|c|}
\hline \multirow{3}{*}{$\begin{array}{l}\text { Cesarean section } \\
(\mathrm{n}=823)^{a}\end{array}$} & \multicolumn{5}{|l|}{ Univariate } & \multicolumn{5}{|c|}{ Multivariate ${ }^{b}$} \\
\hline & \multicolumn{5}{|c|}{$25(\mathrm{OH}) \mathrm{D}(\mathrm{nmol} / \mathrm{L})$} & \multicolumn{5}{|c|}{ 25(OH)D (nmol/L) } \\
\hline & $<25.0$ & $25.0-37.4$ & $37.5-49.9$ & $50.0-74.9$ & $>75.0$ & $<25.0$ & $25.0-37.4$ & $37.5-49.9$ & $50.0-74.9$ & $>75.0$ \\
\hline All women & $\begin{array}{l}1.02 \\
(0.57-1.79)\end{array}$ & $\begin{array}{l}0.88 \\
(0.69-1.12)\end{array}$ & $\begin{array}{l}0.97 \\
(0.76-1.23)\end{array}$ & 1.00 (ref) & $\begin{array}{l}0.77 \\
(0.56-1.08)\end{array}$ & $\begin{array}{l}0.87 \\
(0.48-1.60)\end{array}$ & $\begin{array}{l}0.93 \\
(0.72-1.21)\end{array}$ & $\begin{array}{l}1.06 \\
(0.81-1.37)\end{array}$ & 1.00 (ref) & $\begin{array}{l}0.84 \\
(0.59-1.19)\end{array}$ \\
\hline \multicolumn{11}{|c|}{ Maternal age (years) } \\
\hline$<30$ & $\begin{array}{l}1.23 \\
(0.55-2.76)\end{array}$ & $\begin{array}{l}1.18 \\
(0.83-1.66)\end{array}$ & $\begin{array}{l}1.05 \\
(0.75-1.49)\end{array}$ & 1.00 (ref) & $\begin{array}{l}0.87 \\
(0.53-1.42)\end{array}$ & $\begin{array}{l}1.00 \\
(0.43-2.33)\end{array}$ & $\begin{array}{l}1.15 \\
(0.80-1.65)\end{array}$ & $\begin{array}{l}1.08 \\
(0.75-1.54)\end{array}$ & 1.00 (ref) & $\begin{array}{l}0.95 \\
(0.57-1.58)\end{array}$ \\
\hline $30 \sim$ & $\begin{array}{l}0.92 \\
(0.40-2.09)\end{array}$ & $\begin{array}{l}0.75 \\
(0.52-1.07)\end{array}$ & $\begin{array}{l}1.00 \\
(0.70-1.42)\end{array}$ & 1.00 (ref) & $\begin{array}{l}0.72 \\
(0.45-1.15)\end{array}$ & $\begin{array}{l}0.72 \\
(0.30-1.72)\end{array}$ & $\begin{array}{l}0.74 \\
(0.50-1.08)\end{array}$ & $\begin{array}{l}1.04 \\
(0.71-1.51)\end{array}$ & 1.00 (ref) & $\begin{array}{l}0.75 \\
(0.46-1.23)\end{array}$ \\
\hline \multicolumn{11}{|c|}{ Intrapartum BMI $\left(\mathrm{kg} / \mathrm{m}^{2}\right)$} \\
\hline$<30$ & $\begin{array}{l}0.83 \\
(0.41-1.66)\end{array}$ & $\begin{array}{l}0.89 \\
(0.68-1.17)\end{array}$ & $\begin{array}{l}0.89 \\
(0.68-1.16)\end{array}$ & 1.00 (ref) & $\begin{array}{l}0.77 \\
(0.54-1.11)\end{array}$ & $\begin{array}{l}0.83 \\
(0.40-1.72)\end{array}$ & $\begin{array}{l}0.96 \\
(0.72-1.27)\end{array}$ & $\begin{array}{l}1.01 \\
(0.76-1.34)\end{array}$ & 1.00 (ref) & $\begin{array}{l}0.82 \\
(0.56-1.19)\end{array}$ \\
\hline $30 \sim$ & $\begin{array}{l}1.19 \\
(0.36-3.90)\end{array}$ & $\begin{array}{l}0.91 \\
(0.49-1.68)\end{array}$ & $\begin{array}{l}1.47 \\
(0.79-2.72)\end{array}$ & 1.00 (ref) & $\begin{array}{l}1.17 \\
(0.46-3.01)\end{array}$ & $\begin{array}{l}1.13 \\
(0.33-3.87)\end{array}$ & $\begin{array}{l}0.93 \\
(0.49-1.76)\end{array}$ & $\begin{array}{l}1.56 \\
(0.82-2.94)\end{array}$ & 1.00 (ref) & $\begin{array}{l}1.20 \\
(0.45-3.19)\end{array}$ \\
\hline \multicolumn{11}{|c|}{ Menarche age (years) } \\
\hline$<13$ & $\begin{array}{l}2.26 \\
(0.53-9.63)\end{array}$ & $\begin{array}{l}0.89 \\
(0.49-1.64)\end{array}$ & $\begin{array}{l}1.00 \\
(0.55-1.79)\end{array}$ & 1.00 (ref) & $\begin{array}{l}0.75 \\
(0.35-1.62)\end{array}$ & $\begin{array}{l}1.85 \\
(0.39-8.75)\end{array}$ & $\begin{array}{l}0.98 \\
(0.51-1.91)\end{array}$ & $\begin{array}{l}1.07 \\
(0.57-2.03)\end{array}$ & 1.00 (ref) & $\begin{array}{l}0.89 \\
(0.39-2.02)\end{array}$ \\
\hline $13 \sim$ & $\begin{array}{l}0.70 \\
(0.33-1.52)\end{array}$ & $\begin{array}{l}0.94 \\
(0.69-1.27)\end{array}$ & $\begin{array}{l}0.97 \\
(0.72-1.31)\end{array}$ & 1.00 (ref) & $\begin{array}{l}0.69 \\
(0.45-1.05)\end{array}$ & $\begin{array}{l}0.58 \\
(0.26-1.29)\end{array}$ & $\begin{array}{l}0.99 \\
(0.72-1.37)\end{array}$ & $\begin{array}{l}1.06 \\
(0.77-1.46)\end{array}$ & 1.00 (ref) & $\begin{array}{l}0.70 \\
(0.44-1.10)\end{array}$ \\
\hline $15 \sim$ & $\begin{array}{l}1.59 \\
(0.47-5.47)\end{array}$ & $\begin{array}{l}0.89 \\
(0.50-1.61)\end{array}$ & $\begin{array}{l}1.10 \\
(0.61-1.98)\end{array}$ & 1.00 (ref) & $\begin{array}{l}1.44 \\
(0.64-3.25)\end{array}$ & $\begin{array}{l}1.88 \\
(0.50-7.16)\end{array}$ & $\begin{array}{l}0.95 \\
(0.49-1.84)\end{array}$ & $\begin{array}{l}1.20 \\
(0.61-2.37)\end{array}$ & 1.00 (ref) & $\begin{array}{l}1.56 \\
(0.65-3.75)\end{array}$ \\
\hline \multicolumn{11}{|c|}{ Menstrual cycle (days) } \\
\hline $21 \sim$ & $\begin{array}{l}1.06 \\
(0.58-1.94)\end{array}$ & $\begin{array}{l}1.02 \\
(0.79-1.33)\end{array}$ & $\begin{array}{l}1.01 \\
(0.78-1.31)\end{array}$ & 1.00 (ref) & $\begin{array}{l}0.77 \\
(0.54-1.10)\end{array}$ & $\begin{array}{l}0.87 \\
(0.46-1.66)\end{array}$ & $\begin{array}{l}1.00 \\
(0.76-1.33)\end{array}$ & $\begin{array}{l}1.06 \\
(0.80-1.40)\end{array}$ & 1.00 (ref) & $\begin{array}{l}0.80 \\
(0.55-1.17)\end{array}$ \\
\hline $\begin{array}{l}36 \sim \text { or } \\
\text { irregularity }\end{array}$ & $\begin{array}{l}1.20 \\
(0.19-7.68)\end{array}$ & $\begin{array}{l}0.44 \\
(0.21-0.90)\end{array}$ & $\begin{array}{l}0.86 \\
(0.44-1.68)\end{array}$ & 1.00 (ref) & $\begin{array}{l}1.47 \\
(0.48-4.46)\end{array}$ & $\begin{array}{l}0.77 \\
(0.10-5.80)\end{array}$ & $\begin{array}{l}0.52 \\
(0.23-1.16)\end{array}$ & $\begin{array}{l}0.99 \\
(0.48-2.05)\end{array}$ & 1.00 (ref) & $\begin{array}{l}1.69 \\
(0.49-5.74)\end{array}$ \\
\hline \multicolumn{11}{|c|}{ Abnormal pregnancy history } \\
\hline No & $\begin{array}{l}1.08 \\
(0.55-2.09)\end{array}$ & $\begin{array}{l}0.87 \\
(0.66-1.14)\end{array}$ & $\begin{array}{l}1.00 \\
(0.77-1.31)\end{array}$ & 1.00 (ref) & $\begin{array}{l}0.65 \\
(0.45-0.96)\end{array}$ & $\begin{array}{l}0.90 \\
(0.44-1.84)\end{array}$ & $\begin{array}{l}0.91 \\
(0.68-1.22)\end{array}$ & $\begin{array}{l}1.08 \\
(0.81-1.44)\end{array}$ & 1.00 (ref) & $\begin{array}{l}0.70 \\
(0.47-1.05)\end{array}$ \\
\hline Yes & $\begin{array}{l}0.76 \\
(0.25-2.32)\end{array}$ & $\begin{array}{l}1.06 \\
(0.60-1.87)\end{array}$ & $\begin{array}{l}0.95 \\
(0.54-1.66)\end{array}$ & 1.00 (ref) & $\begin{array}{l}1.90 \\
(0.83-4.36)\end{array}$ & $\begin{array}{l}0.76 \\
(0.23-2.51)\end{array}$ & $\begin{array}{l}1.09 \\
(0.59-2.02)\end{array}$ & $\begin{array}{l}1.06 \\
(0.57-1.97)\end{array}$ & 1.00 (ref) & $\begin{array}{l}2.08 \\
(0.86-5.03)\end{array}$ \\
\hline \multicolumn{11}{|l|}{ Sampling season } \\
\hline Spring & $\begin{array}{l}1.11 \\
(0.50-2.46)\end{array}$ & $\begin{array}{l}0.73 \\
(0.46-1.14)\end{array}$ & $\begin{array}{l}0.77 \\
(0.48-1.23)\end{array}$ & 1.00 (ref) & $\begin{array}{l}0.55 \\
(0.25-1.18)\end{array}$ & $\begin{array}{l}0.98 \\
(0.41-2.31)\end{array}$ & $\begin{array}{l}0.86 \\
(0.52-1.42)\end{array}$ & $\begin{array}{l}0.95 \\
(0.57-1.58)\end{array}$ & 1.00 (ref) & $\begin{array}{l}0.65 \\
(0.28-1.47)\end{array}$ \\
\hline Summer & $\begin{array}{l}0.57 \\
(0.11-3.02)\end{array}$ & $\begin{array}{l}0.90 \\
(0.59-1.39)\end{array}$ & $\begin{array}{l}0.88 \\
(0.58-1.32)\end{array}$ & 1.00 (ref) & $\begin{array}{l}0.58 \\
(0.33-1.01)\end{array}$ & $\begin{array}{l}0.82 \\
(0.14-4.80)\end{array}$ & $\begin{array}{l}0.99 \\
(0.62-1.59)\end{array}$ & $\begin{array}{l}0.95 \\
(0.61-1.49)\end{array}$ & 1.00 (ref) & $\begin{array}{l}0.73 \\
(0.40-1.33)\end{array}$ \\
\hline Autumn & $\begin{array}{l}0.44 \\
(0.08-2.41)\end{array}$ & $\begin{array}{l}1.02 \\
(0.57-1.83)\end{array}$ & $\begin{array}{l}1.14 \\
(0.65-2.01)\end{array}$ & 1.00 (ref) & $\begin{array}{l}1.18 \\
(0.60-2.34)\end{array}$ & $\begin{array}{l}0.46 \\
(0.08-2.75)\end{array}$ & $\begin{array}{l}1.21 \\
(0.63-2.29)\end{array}$ & $\begin{array}{l}1.49 \\
(0.80-2.75)\end{array}$ & 1.00 (ref) & $\begin{array}{l}1.25 \\
(0.60-2.63)\end{array}$ \\
\hline Winter & $\begin{array}{l}1.07 \\
(0.27-4.25)\end{array}$ & $\begin{array}{l}0.86 \\
(0.50-1.48)\end{array}$ & $\begin{array}{l}1.20 \\
(0.70-2.05)\end{array}$ & 1.00 (ref) & $\begin{array}{l}1.04 \\
(0.47-2.34)\end{array}$ & $\begin{array}{l}0.70 \\
(0.16-3.11)\end{array}$ & $\begin{array}{l}0.90 \\
(0.50-1.62)\end{array}$ & $\begin{array}{l}1.20 \\
(0.66-2.18)\end{array}$ & 1.00 (ref) & $\begin{array}{l}1.00 \\
(0.41-2.45)\end{array}$ \\
\hline
\end{tabular}

a All values are OR (95\% CIs); ${ }^{\mathrm{b}}$ The adjustment factors included maternal age, intrapartum BMI, birthplace, parity, menarche age, menstrual cycle, abnormal pregnancy history, sampling season, previously diagnosed hypertension or diabetes, family history of hypertension or diabetes or history of uterine fibroids (excluded the stratified factor in each stratum). Abbreviations: 25(OH)D, 25-hydroxyvitamin D; BMI, body mass index.

\section{Discussion}

Pregnancy is a special stage in the life of women. Their hormone levels and metabolic conditions change and the required nutrients increase significantly. So, in this period, women are prone to vitamin $\mathrm{D}$ deficiency. The requirement for vitamin $\mathrm{D}$ during pregnancy will increase by $4-5$ times to meet the needs of fetal bone growth and extra calcium [17]. Therefore, the lack of vitamin D was prevalent in the world. Shand et al. had completed a prospective cohort study of 221 women in early pregnancy, and they found $75 \%$ of pregnant women with vitamin D insufficiency, $53 \%$ of pregnant women with vitamin D deficiency, and serum vitamin $\mathrm{D}$ levels were significantly lower in Asian women than in Caucasians [18]. Schneuer et al. examined serum $25(\mathrm{OH}) \mathrm{D}$ in 5109 Australia women in the first trimester. They reported the median 25(OH)D concentrations was $56.4 \mathrm{nmol} / \mathrm{L}$, and the serum $25(\mathrm{OH}) \mathrm{D}$ concentrations showed significant variation by parity, smoking, weight, sampling season, country of birth, and socioeconomic status [16]. Recently, in a Belgium nationwide survey, 1311 pregnant women were collected from 55 obstetric clinics, and $74.1 \%$ of them were found to be in a status of vitamin D deficiency [19]. At present, the status of vitamin D deficiency in China is also very serious. Song et al. conducted an investigation involving 125 pregnant women in Beijing, and showed $96.8 \%$ of pregnant women with vitamin $\mathrm{D}$ deficiency, and about half of them with severe lack of vitamin D $[25(\mathrm{OH}) \mathrm{D}<25$ nmol/L] [20]. In our study, only $10.8 \%$ of pregnant women were in a status of adequate vitamin $\mathrm{D}$, about $89.2 \%$ of pregnant women were in a status of vitamin $\mathrm{D}$ deficiency, and $2.8 \%$ of the subjects were severely deficient. Thus, the status of serum vitamin D during pregnancy is not optimistic. Since the vitamin D levels 
during pregnancy are closely related with a series of adverse pregnancy outcomes, the medical workers and pregnant women should pay enough attention to the vitamin D levels.

The constantly improvement of urbanization and modernization resulted in the air pollution, outdoor activities reduction, and the use of anti-ultraviolet products, all of which could lead to insufficient sunshine [21]. Thus, the rate of vitamin D deficiency was significantly increased, especially in pregnant women. This study showed that the serum $25(\mathrm{OH}) \mathrm{D}$ concentrations also have significant variation by sampling seasons. It is relatively high in summer and autumn, and relatively low in spring and winter, which further validated that the vitamin D concentrations was closely associated with the sunlight exposure. In addition, our study found that serum $25(\mathrm{OH}) \mathrm{D}$ concentrations were relatively low in pregnant women with intrapartum $B M I \geq 30 \mathrm{~kg} / \mathrm{m}^{2}$. Vitamin D is lipid soluble vitamin, so it is easy to be absorbed by adipocytes and stored in adipose tissue, which may affect the vitamin D biological effect. Therefore, obesity can also cause blood 25(OH)D concentrations decreased. With the improvement of living standards, the obese population continues to expand, especially for the pregnant women with over-nutrition, which may also lead to vitamin D deficiency in pregnant women.

In this study, the cesarean section rate was relatively high in pregnancy women who were older, who have higher intrapartum BMI, or who have an abnormal pregnancy history, suggesting advanced maternal age, high BMI, adverse pregnancy history may be the risk factors of cesarean section. Compared with 25(OH)D from 50.0 to $74.9 \mathrm{nmol} / \mathrm{L}$, women with low 25(OH)D concentrations ( $<50.0 \mathrm{nmol} / \mathrm{L})$ was not significantly associated with caesarean section. Only in the subgroup of the women without abnormal pregnancy history, higher $25(\mathrm{OH}) \mathrm{D}$ concentrations (> $75.0 \mathrm{nmol} / \mathrm{L}$ ) could significantly decrease the risk of caesarean section. And after adjusting for confounding factors, a borderline significant protective effect was observed for higher $25(\mathrm{OH}) \mathrm{D}$. In total, the serum $25(\mathrm{OH}) \mathrm{D}$ concentrations may not be related with cesarean section. Further studies with large sample size in diverse populations are warranted to validate the association between maternal vitamin D levels and the subsequent delivery mode.

\section{Abbreviations}

25(OH)D: 25-Hydroxyvitamin D; ELISA: euzymelinked immunosorbent assay; ORs: odds ratios; CIs: confidence interval; BMI: body mass index.

\section{Acknowledgement}

This work was supported in part by the National Key Basic Research Program of China (2013CB530604), the National Natural Science Foundation of China (81600685, 81402147), the Natural Science Foundation of Jiangsu Province (BK20160141), the Medical Science and technology development Foundation of Nanjing Department of Health (YKK16201), the Changzhou Natural Science Foundation (CJ20140021), the Jiangsu Provincial Medical Youth Talent (QNRC2016304), and the Science and Technology Development Fund of the Nanjing Medical University (2015NJMUZD062).

\section{Competing Interests}

The authors have declared that no competing interest exists.

\section{References}

1. Burris HH, Rifas-Shiman SL, Camargo CA, Jr., et al. Plasma 25-hydroxyvitamin D during pregnancy and small-for-gestational age in black and white infants. Ann Epidemiol. 2012; 22: 581-6.

2. Ponsonby AL, Lucas RM, Lewis $\mathrm{S}$, et al. Vitamin D status during pregnancy and aspects of offspring health. Nutrients. 2010; 2: 389-407.

3. Bell TD, Demay MB, Burnett-Bowie SA. The biology and pathology of vitamin D control in bone. J Cell Biochem. 2010; 111: 7-13.

4. Lapillonne A. Vitamin D deficiency during pregnancy may impair maternal and fetal outcomes. Med Hypotheses. 2010; 74: 71-5.

5. Nassar N, Halligan GH, Roberts CL, et al. Systematic review of first-trimester vitamin D normative levels and outcomes of pregnancy. Am J Obstet Gynecol. 2011; 205: 208 e1-7.

6. Wen J, Hong $Q$, Zhu L, et al. Association of maternal serum 25-hydroxyvitamin D concentrations in second and third trimester with risk of gestational diabetes and other pregnancy outcomes. Int J Obes (Lond). 2017; 41: 489-96.

7. Holick MF, Binkley NC, Bischoff-Ferrari HA, et al. Evaluation, treatment, and prevention of vitamin D deficiency: an Endocrine Society clinical practice guideline. J Clin Endocrinol Metab. 2011; 96: 1911-30.

8. Hollis BW. Circulating 25-hydroxyvitamin D levels indicative of vitamin D sufficiency: implications for establishing a new effective dietary intake recommendation for vitamin D. J Nutr. 2005; 135: 317-22.

9. Practice ACoO. ACOG Committee Opinion No. 495: Vitamin D: Screening and supplementation during pregnancy. Obstet Gynecol. 2011; 118: 197-8.

10. Cavalier E, Delanaye P, Chapelle JP, et al. Vitamin D: current status and perspectives. Clin Chem Lab Med. 2009; 47: 120-7.

11. Khunpradit $S$, Tavender E, Lumbiganon $P$, et al. Non-clinical interventions for reducing unnecessary caesarean section. Cochrane Database Syst Rev. 2011; 6: CD005528.

12. Yuan C, Gaskins AJ, Blaine AI, et al. Association between cesarean birth and risk of obesity in offspring in childhood, adolescence, and early adulthood. JAMA Pediatr. 2016; 170: e162385

13. Liu S, Liston RM, Joseph KS, et al. Maternal mortality and severe morbidity associated with low-risk planned cesarean delivery versus planned vaginal delivery at term. CMAJ. 2007; 176: 455-60.

14. Merewood A, Mehta SD, Chen TC, et al. Association between vitamin D deficiency and primary cesarean section. J Clin Endocrinol Metab. 2009; 94: 940-5.

15. Bowyer L, Catling-Paull C, Diamond T, et al. Vitamin D, PTH and calcium levels in pregnant women and their neonates. Clin Endocrinol (Oxf). 2009; 70: $372-7$.

16. Schneuer FJ, Roberts CL, Guilbert C, et al. Effects of maternal serum 25-hydroxyvitamin D concentrations in the first trimester on subsequent pregnancy outcomes in an Australian population. Am J Clin Nutr. 2014; 99: 287-95.

17. Brannon PM, Picciano MF. Vitamin D in pregnancy and lactation in humans. Annu Rev Nutr. 2011; 31: 89-115.

18. Shand AW, Nassar N, Von Dadelszen P, et al. Maternal vitamin D status in pregnancy and adverse pregnancy outcomes in a group at high risk for pre-eclampsia. BJOG. 2010; 117: 1593-8.

19. Vandevijvere S, Amsalkhir S, Van Oyen H, et al. High prevalence of vitamin D deficiency in pregnant women: a national cross-sectional survey. PLoS One. 2012; 7: e43868. 
20. Song SJ, Si S, Liu J, et al. Vitamin D status in Chinese pregnant women and their newborns in Beijing and their relationships to birth size. Public Health Nutr. 2013; 16: 687-92.

21. Mithal A, Wahl DA, Bonjour J-P, et al. Global vitamin D status and determinants of hypovitaminosis D. Osteoporos Int. 2009; 20: 1807-20. 